\title{
JOURNAL.RU
}

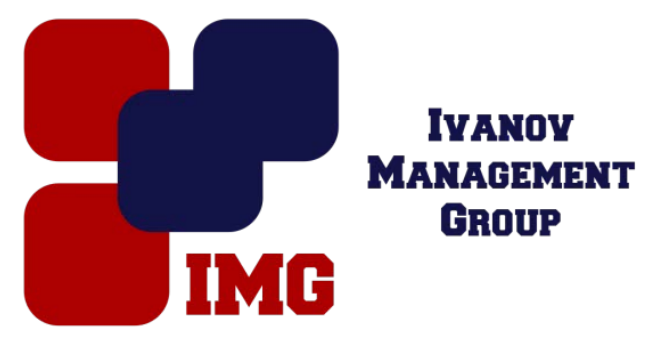

Молочков В.Я., Молочкова И.Д. Дальневосточный государственный технический рыбохозяйственный университет Владивосток, Россия

doi: 10.18411/lj-31-03-2017-2-06

idsp 000001:lj-31-03-2017-2-06

\section{Физические ограничения первичных имерительных преобразователей}

\section{Аннотация}

В статье рассматриваются физические ограничения первичных измерительных преобразователей (датчиков). Приводятся результаты исследования влияния на датчики окружающей среды и расчеты предельных возможностей датчиков, работающих при температуре окружающей среды. Рассматриваются возможности сверхпроводниковых датчиков при исследовании магнитных полей малой интенсивности. Приведенные выкладки могут быть использованы при конструировании различных датчиков.

Ключевые слова: датчики, измерительная техника, физические ограничения.

1. Первичные измерительные преобразователи работающие в нормальных условиях окружающей среды

Существующие первичные измерительные преобразователи (датчики) для измерения различных параметров физических величин основаны на различных физических принципах. Предельные возможности различных датчиков определяются физическими ограничениями.

Аппаратура, построенная с применением датчиков, имеет следующие ограничения:

- связанные с характеристиками измерительной аппаратуры;

- погрешностями метода измерения:

- предельными физическими ограничениями первичных измерительных преобразователей.

Первые два ограничения постоянно преодолеваются по мере совершенствования аппаратуры и методов измерения, третий связан с предельными физическими ограничениями и преодолен быть не может. Он и определяет порог достижимых значений чувствительности и погрешности измеряемой аппаратуры. Ввиду совершенствования современной электронной аппаратуры физические ограничения различных датчиков выходят на передний план. 
Как правило, физические ограничения связаны с самим используемым физическим эффектом датчика - изменением сопротивления, емкости и т.д.

Рассмотрим физические ограничения для датчиков, основанных на различных физических принципах и работающих в диапазоне температур окружающей среды.

Основной вклад в порог чувствительности всех датчиков вносят тепловые флуктуации - тепловые шумы по напряжению, магнитному потоку, емкости, давлению, сопротивлению - в зависимости от типа датчика. Они определяются температурой, при которой они находятся. Поэтому снижение температуры измерительного преобразователя и, при необходимости, температуры элементов измерительной схемы актуальная задача измерения слабых напряжений, токов и магнитных полей.

Шумовая температурная э.д.с. резистивных датчиков имеет значение

$$
e_{u}=\sqrt{4 k T \Delta f R}
$$

где к- постоянная Больцмана, $\mathrm{T}$ - температура, $\Delta \mathrm{f}$ - полоса частот.

Для нормальных условий имеем $e_{u}=1,3 * 10^{-10} \sqrt{\Gamma_{L}^{*} O M}$.

Составляющая от флуктуаций давления - средний квадрат флуктуаций давления от температуры

$$
\overline{P^{2}}=\frac{4 m V P_{0}}{S} d \Delta f,
$$

где $\mathrm{m}$ - масса молекулы, $\mathrm{V}$ - ее скорость, $\mathrm{n}$ - число молекул, $\mathrm{S}$ - площадь, $\Delta \mathrm{f}$ полоса частот.

Для нормальных условий имеем

$$
\sqrt{\frac{--}{P^{2}}}=3,4 * 10^{-6}\left[\text { дин } / \mathrm{cm}^{2} *\right. \text { Гц]. }
$$

Емкостная шумовая составляющая э.д.с. датчиков на последовательно соединенном с емкостью сопротивлении равна

$$
e_{u}=\frac{4 k T R \Delta f}{1+\omega^{2} R^{2} C^{2}},
$$

где $\mathrm{C}$ - емкость, $\mathrm{R}$ - последовательно соединенное с емкостью сопротивление, ш - угловая частота ( $2 \pi \mathrm{f})$, остальные обозначения соответствуют вышеприведенным.

Расчет показывает, что для частоты 100 Гц в полосе 1 Гц, сопротивлении согласованном с сопротивлением емкости и равном 100 Мом, емкости 50 пФ шумовая составляющая достигает $4,1 * 10-9$ В.

\section{2. Сверхпроводниковые первичные измерительные преобразователи}

Одними из информативных параметров при исследовании физических полей Океана являются характеристики магнитного поля - индукция, магнитный поток и их градиенты. Параметры магнитного поля тесно связаны с процессами в Океане - течениями, волнениями, характеристиками дна, которые могут исследоваться по их величинам и градиентам. Однако величина магнитных полей от этих источников чрезвычайно мала, маскируется естественным земным магнитным полем и требует специально разработанных, высокочувствительных 
измерительных устройств. Наиболее приемлемыми первичными измерительными преобразователями являются сверхпроводниковые.

Рассмотрим предельные возможности одного из таких первичных измерительных преобразователей.

Для сверхпроводникового измерительного преобразователя - “сквида" (рисунок 1), состоящего из сверхпроводникового контура 1, охватывающего не сверхпроводниковую площадь Sнс, и двух мостиковых переходов Джозефсона 3 , имеет смысл учитывать шумовые составляющие по э.д.с., и магнитному потоку.

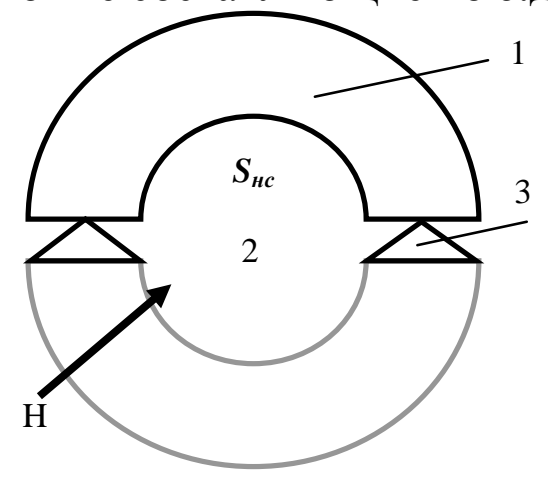

Рисунок 1

Шумовая составляющая магнитного потока для сверхпроводникового измерителя "сквида" может быть рассчитана следующим образом. Магнитный поток существует в площади, охватываемой сверхпроводниковым контуром, дискретно с дискретностью равным кванту магнитного потока. Квант магнитного потока рассчитывается исходя из условия Зоммерфельда-Бора

$$
\oint \overline{p d} s=\oint 2 m v d s \bar{s}+\oint 2 e \bar{A} d s=n h,
$$

где $\mathrm{p}=2 \mathrm{mv}+2 \mathrm{eA}-$ канонический импульс куперовской пары в сверхпроводнике, $\mathrm{m}, \mathrm{e}$ - масса и заряд электрона, V - скорость, A - векторный потенциал, $\mathrm{h}$ постоянная Планка, $\mathrm{n}$ - целое число.

Примем во внимание, что первая составляющая этого уравнения пропорциональна току в слое равном глубине проникновения тока в сверхпроводник. Глубина проникновения магнитного поля в сверхпроводник обычно на порядки меньше толщины контура сверхпроводникового датчика. Выбирая контур квантования, охватывающий внутреннюю площадь за пределами глубины проникновения тока в сверхпроводник, эту составляющую можно исключить.

Вторая составляющая представляет собой магнитный поток внутри несверхпроводящей области охватываемой сверхпроводниковым контуром. Учитывая вышесказанное уравнение можно привести к виду

$$
\Phi=\frac{\mathrm{nh}}{2 \mathrm{e}}=n \Phi_{0} .
$$

Следовательно, в не сверхпроводниковой площади, охватываемой сверхпроводниковым контуром, магнитный поток квантуется с дискретностью магнитного потока равном $\mathrm{h} / 2 \mathrm{e}(2 * 10-15 \mathrm{B \sigma})$. Такими дискретными порциями поток проникает в не сверхпроводниковую площадь, охваченную 
сверхпроводником. Дополнение до кванта потока компенсируется магнитным потоком циркулирующего в сверхпроводниковом контуре тока.

Джозефсоновский переход имеет отличное от нуля падение напряжения при протекании через него тока дополняющего своим полем магнитное поле в площади, охваченной сверхпроводником, до целого числа квантов магнитного потока. Этот ток имеет величину $I_{n}^{2}=k T / L$ и является источником шумового тока, средний квадрат которого равен

$$
I_{n}^{2}=4 k T \Delta f /(2 R)
$$

Его значение является источником шумового магнитного потока величиной

$$
L^{2} I_{n}^{2}=2 L^{2} k T \Delta f / R \text {. }
$$

Отсюда разрешение по потоку при соотношении сигнал/шум равном 1 составит $\Delta \Phi=\left(2 \mathrm{~L}^{2} k T \Delta f / \Phi_{0}^{2} / R\right)^{1 / 2}$.

Контроль циркулирующего в сверхпроводниковом контуре тока возможен по падению напряжения на мостиковых переходах Джозефсона существующего в виде осцилляций напряжения. Измерение этого напряжения позволяет измерять магнитный поток равный долям кванта магнитного потока. В этом случае разрешение ограничивается тепловыми шумами э.д.с. на сопротивлении и тепловым шумами магнитного потока.

При температурах жидкого гелия 4,2 К, индуктивности измерительного контура “сквида" 10-9 Гн и сопротивлении перехода единицы Ом, разрешение доли кванта потока составит $2,5 * 10^{-6} \sqrt[-2]{\Gamma_{L}}$, что в пересчете на магнитный поток составит 2*10-18Вб. Это на три порядка большая чувствительность, чем естественные локальные вариации земного поля.

Для наблюдения квантования магнитного потока необходимо выполнять условие $\Phi_{m}^{2} \angle\left(\Phi_{0} / 2\right)^{2}$, т.е. чтобы средне-квадратичное значение шумового потока было меньше средне-квадратичного значения полукванта потока в площади охватываемой сверхпроводником.

Реальный “сквид" должен иметь как можно меньшее значение индуктивности контура - идеальное значение нулевое, однако реальная конструкция "сквида" имеет, естественно, конечное значение индуктивности. Максимально допустимая индуктивность контура “сквида” может быть рассчитана, исходя из значения флуктуационного тока, нарушающего когерентные свойства датчика, при которых перестает наблюдаться квантование магнитного потока. Для расчета этого значения индуктивности приравняем магнитную энергию флуктуационного тока $\frac{\mathrm{LI}^{2}}{2}$ к тепловой энергии kT и определим значение индуктивности предельного максимального значения индуктивности контура “сквида"

$$
L_{\max }=\frac{h^{2}}{8 e k T}
$$


где е - заряд электрона, остальные величины определены выше. Для температуры $\mathrm{T}=4,2 \mathrm{~K}$ это значение равно 10-8 Гн.

Таким образом, рассчитанные характеристики определяют предельные значения на уровне физических ограничений и в совокупности с приведенными реальными параметрами контуров “сквида" являются техническим заданием при разработке “сквидов” различных типов и конструкций.

Можно сделать заключение, что “сквид” является приемлемым и в ряде случаев единственно приемлемым по чувствительности устройством для измерения магнитных полей различных объектов, в том числе физических полей Океана. Градиентометрическая разновидность “сквида ”, имеющая измерительную базу около 1 см, позволяет исследовать изменения магнитных полей, связанных с процессами в Океане, на фоне флуктуаций магнитного поля Земли.

\section{Литература}

1. Split Many Mode Methods for Window Josephson Junctions, (with E. Moulit-sas, Y. Gaididei, N. Flytzanis and J.G. Caputo). Int. J. Modern Phys $\neg$ ics C, 9, (1989) 301-324.

2. Молочков В.Я, Есипенко Е.И., Герасимов В.А. Отчет по НИР «Исследование магнитных полей от гидродинамических источников измерителем на основе эффекта Джозефсона», Отчет НИР № гос.рег. 01850039574, 1990 